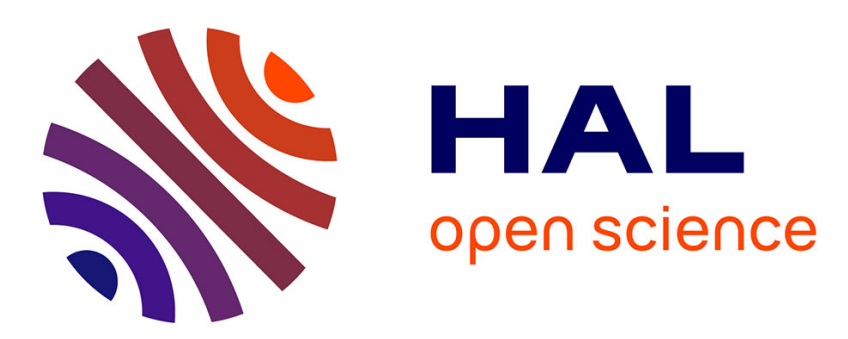

\title{
Ramsey for complete graphs with a dropped edge or a triangle
}

Luis Montejano, Jonathan Chappelon, Jorge Luis Ramírez Alfonsín

\section{To cite this version:}

Luis Montejano, Jonathan Chappelon, Jorge Luis Ramírez Alfonsín. Ramsey for complete graphs with a dropped edge or a triangle. Electronic Notes in Discrete Mathematics, 2017, LAGOS'17 - IX Latin and American Algorithms, Graphs and Optimization, 62, pp.21-25. 10.1016/j.endm.2017.10.005 . hal-02053333

\section{HAL Id: hal-02053333 \\ https://hal.science/hal-02053333}

Submitted on 1 Mar 2019

HAL is a multi-disciplinary open access archive for the deposit and dissemination of scientific research documents, whether they are published or not. The documents may come from teaching and research institutions in France or abroad, or from public or private research centers.
L'archive ouverte pluridisciplinaire HAL, est destinée au dépôt et à la diffusion de documents scientifiques de niveau recherche, publiés ou non, émanant des établissements d'enseignement et de recherche français ou étrangers, des laboratoires publics ou privés. 


\title{
Ramsey for complete graphs with a dropped edge or a triangle
}

\author{
Luis Pedro Montejano ${ }^{1}$ \\ Centro de Investigación en Matemáticas \\ CIMAT \\ Guanajuato, México
}

\author{
Jonathan Chappelon and Jorge Luis Ramírez Alfonsín 2,3 \\ Institut de Mathématiques et de Modélisation de Montpellier \\ Université de Montpellier \\ Montpellier, France
}

\begin{abstract}
Let $K_{[k, t]}$ be the complete graph on $k$ vertices from which a set of edges, induced by a clique of order $t$, has been dropped (note that $K_{[k, 1]}$ is just $K_{k}$ ). In this paper we study $R\left(K_{\left[k_{1}, t_{1}\right]}, \ldots, K_{\left[k_{r}, t_{r}\right]}\right)$ (the smallest integer $n$ such that for any $r$-edge coloring of $K_{n}$ there always occurs a monochromatic $K_{\left[k_{i}, t_{i}\right]}$ for some $i$ ).

We first present a general upper bound (containing the well-known Graham-Rödl upper bound for complete graphs in the particular case when $t_{i}=1$ for all $i$ ). We then focus our attention when $r=2$ and dropped cliques of order 2 and 3 (edges and triangles). We give the exact value for $R\left(K_{[n, 2]}, K_{[4,3]}\right)$ and $R\left(K_{[n, 3]}, K_{[4,3]}\right)$ for all $n \geq 2$.
\end{abstract}

Keywords: Ramsey number, recursive formula. 


\section{Introduction}

Let $K_{n}$ be a complete graph and let $r \geq 2$ be an integer. A $r$-edge coloring of a graph is a surjection from $E(G)$ to $\{0, \ldots, r-1\}$ (and thus each color class is not empty). Let $k \geq t \geq 1$ be positive integers. We denote by $K_{[k, t]}$ the complete graph on $k$ vertices from which a set of edges, induced by a clique of order $t$, has been dropped, see Figure 1.

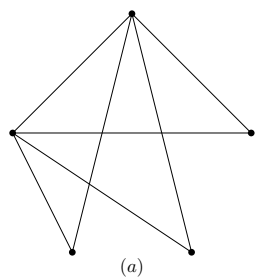

(a)

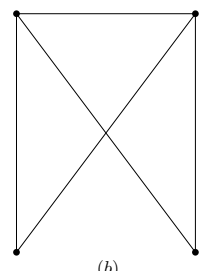

(b)

Fig. 1. (a) $K_{[5,3]}$ and (b) $K_{[4,2]}$

Let $k_{1}, \ldots, k_{r}$ and $t_{1}, \ldots, t_{r}$ be positive integers with $k_{i} \geq t_{i}$ for all $i \in$ $\{1, \ldots, r\}$. Let $R\left(\left[k_{1}, t_{1}\right], \ldots,\left[k_{r}, t_{r}\right]\right)$ be the smallest integer $n$ such that for any $r$-edge coloring of $K_{n}$ there always occurs a monochromatic $K_{\left[k_{i}, t_{i}\right]}$ for some $i$. In the case when $k_{i}=t_{i}$ for some $i$, we set

$$
R\left(\left[k_{1}, t_{1}\right], \ldots,\left[k_{i-1}, t_{i-1}\right],\left[t_{i}, t_{i}\right],\left[k_{i+1}, t_{i+1}\right], \ldots,\left[k_{r}, t_{r}\right]\right) \leq t_{i} .
$$

We note that equality is reached at $\min _{1 \leq i \leq r}\left\{t_{i} \mid t_{i}=k_{i}\right\}$. Since the set of all the edges of $K_{\left[t_{i}, t_{i}\right]}$ (which is empty) can always be colored with color $i$. We also notice that the case $R\left(\left[k_{1}, 1\right], \ldots,\left[k_{r}, 1\right]\right)$ is exactly the classical Ramsey number $r\left(k_{1}, \ldots, k_{r}\right)$ (the smallest integer $n$ such that for any $r$-edge coloring of $K_{n}$ there always occurs a monochromatic $K_{k_{i}}$ for some $i$ ). We refer the reader to the excellent survey [6] on Ramsey numbers for small values. In this paper, we investigate $R\left(\left[k_{1}, t_{1}\right], \ldots,\left[k_{r}, t_{r}\right]\right)$.

\section{General upper bound}

In this section we present a recursive formula (Lemma 2.1) that yields to an explicit general upper bound (Theorem 2.2). The latter contains the wellknown explicit general upper bound for $R\left(\left[k_{1}, 1\right], \ldots,\left[k_{r}, 1\right]\right)$ due to Graham and Rödl [3] (see Equation (4)).

\footnotetext{
1 Email: lmontejano@cimat.mx

2 Email: jramirez@um2.fr

3 Email: jonathan.chappelon@um2.fr
} 
The following recursive inequality is classical in Ramsey theory (1) $r\left(k_{1}, k_{2}, \ldots, k_{r}\right) \leq r\left(k_{1}-1, k_{2}, \ldots, k_{r}\right)+r\left(k_{1}, k_{2}-1, \ldots, k_{r}\right)+\cdots+$ $+r\left(k_{1}, k_{2}, \ldots, k_{r}-1\right)-(r-2)$

In the same spirit, we have the following.

Lemma 2.1 Let $r \geq 2$ and let $k_{1}, \ldots, k_{r}$ and $t_{1}, \ldots, t_{r}$ be positive integers with $k_{i} \geq t_{i}+1 \geq 2$ for all $i$. Then,

$$
\begin{aligned}
R\left(\left[k_{1}, t_{1}\right], \ldots,\left[k_{r}, t_{r}\right]\right) \leq & R\left(\left[k_{1}-1, t_{1}\right],\left[k_{2}, t_{2}\right], \ldots,\left[k_{r}, t_{r}\right]\right) \\
& +R\left(\left[k_{1}, t_{1}\right],\left[k_{2}-1, t_{2}\right], \ldots,\left[k_{r}, t_{r}\right]\right) \\
& \vdots \\
& +R\left(\left[k_{1}, t_{1}\right],\left[k_{2}, t_{2}\right], \ldots,\left[k_{r}-1, t_{r}\right]\right)-(r-2) .
\end{aligned}
$$

A similar recursive inequality has been treated in [7] in a much more general setting in which a family of graphs are intrinsically constructed via two operations disjoin unions and joins (see also [4] for the case $r=2$ ). However, it is not clear how the latter could be used to obtain Lemma 2.1 that allows us to give the following general upper bound for $R\left(\left[k_{1}, t_{1}\right], \ldots,\left[k_{r}, t_{r}\right]\right.$ ) (which was not considered in [7]).

Theorem 2.2 Let $r \geq 2$ be a positive integer and let $k_{1}, \ldots, k_{r}$ and $t_{1}, \ldots, t_{r}$ be positive integers such that $k_{i} \geq t_{i}$ for all $i \in\{1, \ldots, r\}$. Then,

$$
R\left(\left[k_{1}, t_{1}\right], \ldots,\left[k_{r}, t_{r}\right]\right) \leq \max _{1 \leq i \leq r}\left\{t_{i}\right\}\left(\begin{array}{l}
k_{1}+\cdots+k_{r}-\left(t_{1}+\cdots+t_{r}\right) \\
k_{1}-t_{1}, k_{2}-t_{2}, \ldots+k_{r}-t_{r}
\end{array}\right)
$$

where $\left(\begin{array}{c}n_{1}+n_{2}+\cdots+n_{r} \\ n_{1}, n_{2}, \ldots \ldots, n_{r}\end{array}\right)$ is the multinomial coefficient defined by $\left(\begin{array}{c}n_{1}+n_{2}+\cdots+n_{r} \\ n_{1}, n_{2}, \ldots \ldots, n_{r}\end{array}\right)=$ $\frac{\left(n_{1}+\cdots+n_{r}\right) !}{n_{1} ! n_{2} ! \cdots n_{r} !}$, for all nonnegative integers $n_{1}, \ldots, n_{r}$.

Theorem 2.2 is a natural generalization of the well-known explicit upper bound for classical Ramsey numbers. Indeed, an immediate consequence of Theorem 2.2 (by taking $t_{i}=1$ for all $i$ ) is the following classical upper bound due to Graham and Rödl [3, (2.48)]

$$
R\left(\left[k_{1}, 1\right], \ldots,\left[k_{r}, 1\right]\right) \leq\left(\begin{array}{c}
k_{1}+\cdots+k_{r}-r \\
k_{1}-1, \ldots, k_{r}-1
\end{array}\right) .
$$

Let $k \geq t \geq 2$ and $r \geq 2$ be integers and let $R_{r}([k, t])=R(\underbrace{[k, t], \ldots,[k, t]}_{r})$. An immediate consequence of Theorem 2.2 (by taking $k=k_{1}=\cdots=k_{n}$ and 
$\left.t=t_{1}=\cdots=t_{n}\right)$ is the following inequality

$$
R_{r}([k, t]) \leq t\left(\begin{array}{c}
r(k-t) \\
k-t, \ldots, k-t
\end{array}\right)
$$

Moreover, if $t=1$ then

$$
R_{r}([k, 1]) \leq \frac{(r k-r) !}{((k-1) !)^{r}}
$$

\section{Exact values}

By the so-called Chvátal's result [2], we know that the exact value of the Ramsey number of $K_{[4,3]}$ (a star) versus cliques is given by $R([n, 1],[4,3])=3 n-2$ for all $n \geq 1$. We then naturally focus our attention to the Ramsey number of $K_{[4,3]}$ versus cliques with either a dropped edge or a dropped triangle, see [1] where $R([m, 1],[n, 2])$ has been computed for numerous cases. We provide the new following exact values of Ramsey numbers.

Theorem 3.1 Let $n \geq 2$ be an integer. Then,

- $R([n, 2],[4,3])=2$ for $n=2$,

- $R([n, 2],[4,3])=5$ for $n=3$,

- $R([n, 2],[4,3])=3 n-5$ for $n \geq 4$.

Theorem 3.2 Let $n \geq 2$ be an integer. Then,

- $R([n, 3],[4,3])=3$ for $n=3$,

- $R([n, 3],[4,3])=6$ for $n=4$,

- $R([n, 3],[4,3])=8$ for $n=5$,

- $R([n, 3],[4,3])=11$ for $n=6$,

- $R([n, 3],[4,3])=3 n-8$ for $n \geq 7$.

3.1 An estimation for $R([n, 2],[5,3])$

By considering $K_{[5,3]}$ as the book graph $\mathrm{B}_{3}$, it was proved in $[5,8]$ that

$$
R([n, 1],[5,3]) \leq \frac{3 n^{2}}{\log (n / e)},
$$

for all positive integers $n$.

The following result is a first estimation for the value $R([n, 2],[5,3])$.

Theorem 3.3 Let $n \geq 2$ be an integer. Then, 
- $R([n, 2],[5,3])=2$ for $n=2$,

- $R([n, 2],[5,3])=7$ for $n=3$,

- $R([n, 2],[5,3]) \leq 3\left(\begin{array}{c}n+1 \\ 2\end{array}\right)-5 n+4$ for $n \geq 4$.

\section{References}

[1] J. Chappelon, L.P. Montejano and J.L. Ramírez Alfonsín, On Ramsey numbers of complete graphs with dropped stars, Discrete Applied Math. 210 (2016), 200206.

[2] V. Chvátal, Tree-complete Ramsey numbers, J. Graph Theory 1 (1977), 93.

[3] R. Graham and V. Rödl, Numbers in Ramsey theory, Surveys in Combinatorics 1987, 123, London Mathematics Society Lecture Note Series (1987) 111-153.

[4] Y.R. Huang, K. Zhang, New upper bounds for Ramsey numbers, European J. Combin. 19(3) (1998), 391-394.

[5] Y. Li, C.C. Rousseau, On Book-Complete Graph Ramsey Numbers, J. Combin. Theory Ser. B 68 (1996), 36-44.

[6] S.P. Radziszowski, Small Ramsey numbers, Electron. J. Combin. 1 (1994), Dynamic Survey 1, 30 pp (electronic) (revision \#14 January 12, 2014).

[7] L. Shi, K. Zhang, A bound for multicolor Ramsey numbers, Discrete Math. 226(1-3) (2001), 419-421.

[8] B. Sudakov, Large $K_{r}$-Free Subgraphs in $K_{s}$-Free Graphs abd Some Other Ramsey-Type Problems, Random Structures and Algorithm 26 (2005), 253-265. 